\title{
Is the ideal mother a sensitive mother? Beliefs about early childhood parenting in mothers across the globe
}

\author{
Judi Mesman,' Marinus van IJzendoorn,' Kazuko Behrens, ${ }^{2}$ \\ Olga Alicia Carbonell, ${ }^{3}$ Rodrigo Cárcamo, ${ }^{1,4}$ \\ Inbar Cohen-Paraira, ${ }^{5}$ Christian de la Harpe, ${ }^{6}$ Hatice Ekmekçi, ${ }^{1}$ \\ Rosanneke Emmen,' Jailan Heidar,' Kiyomi Kondo-lkemura,' \\ Cindy Mels, ${ }^{8}$ Haatembo Mooya, ${ }^{9}$ Sylvia Murtisari, ${ }^{10}$ \\ Magaly Nóblega, " Jenny Amanda Ortiz, ${ }^{12}$ \\ Abraham Sagi-Schwartz, ${ }^{5}$ Francis Sichimba, ${ }^{9}$ Isabel Soares, ${ }^{13}$ \\ Howard Steele, ${ }^{14}$ Miriam Steele, ${ }^{14}$ Marloes Pape, \\ Joost van Ginkel,' 'René van der Veer,' Lamei Wang, ${ }^{15}$ \\ Bilge Selcuk, ${ }^{16}$ Melis Yavuz, ${ }^{16}$ and Ghadir Zreik ${ }^{5}$
}

\begin{abstract}
In this article, we test the hypothesis that beliefs about the ideal mother are convergent across cultures and that these beliefs overlap considerably with attachment theory's notion of the sensitive mother. In a sample including 26 cultural groups from 15 countries around the globe, 75I mothers sorted the Maternal Behavior Q-Set to reflect their ideas about the ideal mother. The results show strong convergence between maternal beliefs about the ideal mother and attachment theory's description of the sensitive mother across groups. Cultural group membership significantly predicted variations in maternal sensitivity belief scores, but this effect was substantially accounted for by group variations in socio-demographic factors. Mothers living in rural versus urban areas, with a low family income, and with more children, were less likely to describe the ideal mother as highly sensitive. Cultural group membership did remain a significant predictor of variations in maternal sensitivity belief scores above and beyond socio-demographic predictors. The findings are discussed in terms of the universal and culture-specific aspects of the sensitivity construct.
\end{abstract}

\section{Keywords}

cross-culture, maternal sensitivity, mother-infant relationships, socioeconomic status

Attachment theory was formulated to represent a universally applicable account of the bond between caregivers and infants based on evolutionary and ethological considerations (Bowlby, 1969). Although the number of cross-cultural studies is still limited, empirical research indeed provides some support for the universality of the major tenets of attachment theory, with evidence for the universality hypothesis that across the world, (virtually) all infants become attached to one or more specific caregivers, and the normativity hypothesis that secure attachment is the most common form of attachment across cultures (Mesman, Van IJzendoorn, \& SagiSchwartz, in press). In addition, there is evidence that maternal beliefs about the ideal child overlap considerably with the notion of secure-base behavior and show high agreement across cultures (Posada et al., 1995, 2013; see also Sternberg \& Lamb, 1992). Cross-cultural research on the tenets of attachment theory has mostly focused on child behaviors, and less attention has been paid to the parental side of the attachment coin. Sensitive parenting is defined as a caregiver's ability to perceive child signals, to interpret these signals correctly, and to respond to them contingently and appropriately (Ainsworth, Bell, \& Stayton, 1974), and has been clearly identified

\footnotetext{
I Centre for Child and Family Studies, Leiden University, the Netherlands

${ }^{2}$ State University of New York, Albany, NY, USA

${ }^{3}$ Pontificia Universidad Javeriana, Bogota, Colombia

${ }^{4}$ University of Magallanes, Punta Arenas, Chile

${ }^{5}$ University of Haifa, Israel

${ }^{6}$ Catholic University of Temuco, Temuco, Araucania, Chile

${ }^{7}$ Tokyo University, Japan

${ }^{8}$ Catholic University of Uruguay, Montevideo, Uruguay

${ }^{9}$ University of Zambia, Lusaka, Zambia

10 Sanata Dharma University, Daerah Istimewa Yogyakarta, Indonesia

1 Pontificia Universidad Católica del Perú, Lima, Peru

12 Universidade Federal do Rio Grande do Sul, Porto Alegre - RS, Brazil

13 University of Minho, Guimaraes, Portugal

14 The New School for Social Research, New York, NY, USA

${ }^{15}$ Chinese Academy of Sciences, Beijing, China

${ }^{16}$ Koc University, Sarıyer/istanbul, Turkey
}

\section{Corresponding author:}

Judi Mesman, Centre for Child and Family Studies, Leiden University, Wassenaarseweg 52, 2333 AK, Leiden, the Netherlands.

Email: mesmanj@fsw.leidenuniv.nl 
as an important parental characteristic that facilitates secure attachment in children (Bakermans-Kranenburg, Van IJzendoorn, \& Juffer, 2003). In the current study that includes 751 mothers from 26 cultural groups in 15 countries, we test the hypothesis that maternal beliefs about the ideal mother converge considerably with the notion of the highly sensitive mother, and we test socio-demographic and cultural predictors of individual variations in this convergence.

Culture can be broadly defined as patterns of behaviors, knowledge, and beliefs acquired through socialization processes, and that distinguishes one group from another group (e.g., Boyd \& Richerson, 2005). Two of the most commonly-used distinctions in the literature refer to individualism versus collectivism (Oyserman, Coon, \& Kemmelmeier, 2002), and autonomous versus relatedness cultural orientation (Kagitcibasi, 2007). In individualistic and autonomous cultures (generally found in urban Western areas), the individual is valued over the group, and parenting is geared towards fostering psychological independence and individual achievement, and is characterized by a distal parenting style and face-to-face contact rather than physical proximity (Feldman \& Masalha, 2010; Keller et al., 2009). In collectivistic cultures and cultures emphasizing relatedness (generally found in non-Western rural areas), the group is valued over the individual, and parenting focuses on fostering social relations and hierarchies and stimulating obedience to group norms, and is characterized by a proximal parenting style expressed by physical closeness (e.g., Keller et al., 2009). A blend of these two broad cultural orientations is described by the autonomous-related cultural model, which characterizes urbanized or migrated groups that originally come from collectivistic cultural backgrounds, but now reside in a more individualistic context (Kagitcibasi, 2007).

It has been argued that the definitions of the main attachmentrelated constructs, including secure attachment and sensitivity, are biased towards individualistic cultural contexts with distal parenting patterns because of their focus on meeting the individual infant's needs and fostering psychological autonomy (e.g., Keller, 2013; Rothbaum, Weisz, Pott, Miyake, \& Morelli, 2000). However, Mary Ainsworth's first descriptions of behaviors that reflect sensitive responsiveness were based on her observations in Uganda (Ainsworth, 1967). Cross-cultural research indeed confirms that sensitivity and attachment can be validly measured in nonWestern contexts, and also relates to secure infant attachment in the expected directions in countries such as China (Ding, Xiu, Wang, Li, \& Wang, 2012), Japan (Vereijken, Riksen-Walraven, \& Kondo-Ikemura, 1997), South Korea (Jin, Jacobvitz, Hazen, \& Jung, 2012), Mali (True, Pisani, \& Oumar, 2001), Mexico (Gojman et al., 2012), and South Africa (Tomlinson, Cooper, \& Murray, 2005). Further, some of the main components of sensitivity represent universally important aspects of caregiving. Availability and proximity are crucial to infant survival in that the child is kept safe. Prompt responding serves the child's common human ability to detect contingencies between one's own behavior and environmental events, and child-centered responsiveness is further conducive of child well-being in that it will get fed when signaling hunger, protected when signaling fear, and cared for when signaling pain.

One way of addressing the universality versus culture-specificity of core attachment concepts is to examine parental beliefs (or ethnotheories) about these concepts in different cultures. Posada and colleagues (1995) used the Attachment Q-Set (AQS) to assess maternal beliefs about ideal child behavior and found that these were similar across seven Western and non-Western countries and overlapped considerably with attachment theory's notion of the secure-base phenomenon (i.e., a child staying close to caregiver in unfamiliar situations, easily comforted by caregiver, and explorative when put at ease). In a recent study (Emmen, Malda, Mesman, Ekmekci, \& Van IJzendoorn, 2012), the Maternal Behavior Q-Set (MBQS; Pederson \& Moran, 1995) was used to assess maternal beliefs about the ideal mother in relation to the sensitivity construct in three different ethnic groups within the Netherlands (majority Dutch, minority Turkish and Moroccan with an immigrant background). The results showed strong convergence between maternal views of the ideal mother and attachment theory's notion of sensitive parenting across cultural groups (Emmen et al., 2012). However, the cultural groups in this study all resided within the same country, and the minority groups represented mostly secondgeneration immigrants who were born in the Netherlands. Thus, replication and extension of this study including a wider range of countries and cultural groups is needed.

As discussed above, potential cultural influences on beliefs about sensitive parenting may center around the individualismcollectivism distinction, and beliefs of parents with individualistic values converging more with the idea of sensitivity than beliefs of parents with collectivistic values (Feldman \& Masahla, 2010; Keller, 2013). Further, a horizontal cultural orientation refers to equality in relations and a vertical orientation refers to social hierarchies (Singelis, Triandis, Bhawuk, \& Gelfand, 1995). Research has shown that vertical but not horizontal orientations within individualism and collectivism were related to authoritarian parenting (Georgiou, Fousiani, Michaelides, \& Stavrinides, 2013). Conversely, horizontal but not vertical orientations have been found to be associated with higher autonomy support (Chirkov, Ryan, \& Willness, 2005). These findings suggest that horizontal rather than vertical orientations may be more conducive to sensitive parenting. Another important aspect of culture is religion. Research regarding religiosity and parenting to date have yielded inconsistent results and is dominated by Western Christian samples (e.g., Mahoney, Pargament, Tarakeshwar, \& Swank, 2001; Vermeer, 2011). In general, the use of religion as a guideline in parenting would be expected to potentially lead to less flexible responsiveness, because fixed factors other than the child's needs play a role in deciding the response. In the Emmen et al. (2012) study using the MBQS to assess maternal beliefs about sensitivity, higher religiosity in childrearing was indeed related to lower convergence between the mothers' beliefs about the ideal mother and the profile of the highly sensitive mother.

There is some evidence that socio-demographic factors are more salient than cultural factors in predicting sensitive parenting (Mesman, Van IJzendoorn, \& Bakermans-Kranenburg, 2012). Parents with fewer years of formal education and parents with a low income have been found to have less favorable attitudes about parenting in general (e.g., Clément \& Chamberland, 2009; Pinderhughes, Bates, Dodge, Pettit, \& Zelli, 2000), sensitivity in particular (Emmen et al., 2012), and show a lower quality of actual parenting behaviors (e.g., Mesman et al., 2012). Parents experiencing socioeconomic hardship may be less inclined to hold views of parenting that require substantial emotional and time investment (such as sensitive parenting), because they are more focused on problems of survival. This may lead these parents to view and experience parenting more as the stressful day-to-day managing of children, which is then more likely to foster parenting beliefs that emphasize the importance of (physical) control rather than beliefs focusing on the importance of warmth and responsiveness (McLoyd, 1998; Pinderhughes et al., 2000). Thus, we would expect higher income and more formal education to relate to maternal beliefs about parenting that converge with the idea of sensitivity. 
Other demographic variables of interest include maternal age, family size, and urban versus rural residence, with evidence to date pointing towards less favorable parenting attitudes and behaviors in younger mothers (e.g., Schlomer \& Belsky, 2013), families with a higher number of children (Furman \& Lanthier, 2002), and families in rural areas (Bornstein et al., 2012).

In the current study, we investigate the cross-cultural applicability of the sensitivity construct in a sample of 751 mothers from 15 countries with a total of 26 cultural groups. The following hypotheses were tested: (1) There is strong convergence between maternal descriptions of the ideal mother and attachment theory's description of the highly sensitive mother across cultural groups, similar to findings regarding the overlap between maternal beliefs about the ideal child and the attachment theory's notion of a securely attached child as described by Posada et al. (1995). (2) Convergence between maternal descriptions of the ideal mother and descriptions of the highly sensitive mother are predicted by socio-demographic variables. More specifically, the extent to which mothers describe the ideal mother as a sensitive mother is expected to show positive associations with family income, maternal education, maternal age, and urbanity of the setting, and a negative association with number of children. (3) Convergence between maternal descriptions of the ideal mother and descriptions of the highly sensitive mother are predicted by cultural variables. More specifically, the extent to which mothers describe the ideal mother as a sensitive mother is expected to show positive associations with individualism and a horizontal cultural orientation, and a negative association with collectivism, a vertical cultural orientation, and emphasis on religion in parenting.

\section{Method}

\section{Sample and procedure}

A total of 751 participants were recruited from 15 different countries representing 26 cultural groups. The countries include (in alphabetical order): Brazil, Chile, China, Colombia, Egypt, Indonesia, Israel, Japan, the Netherlands, Peru, Portugal, Turkey, the United States, Uruguay, and Zambia. Cultural groups within countries were defined by the local research teams based on their knowledge of each group's cultural characteristics. Six countries included multiple cultural groups (Chile, Israel, the Netherlands, Peru, Portugal, and the US). All participants were selected for having at least one child between the ages of 6 months and 6 years. Exclusion criteria were a target child with a severe mental or physical disability, and maternal illiteracy. Recruitment strategies varied across countries, but generally represented convenience sampling through the researchers' networks followed by snowballing, or recruitment of participants from previous or ongoing other studies. All participants received the same information brochure (translated into all relevant languages), and signed the same informed consent form. The brochure informed participants about the international nature of the study and the research goal of examining differences and similarities about maternal beliefs about parenting between countries. Small gifts (monetary or otherwise) as a token of appreciation for participation were adapted to local customs. Socio-demographic and cultural sample characteristics are shown in Table 1.

\section{Measures}

Translation from English into the relevant languages and backtranslation were done by fluent speakers of these languages.
Differences between the English original and the back-translation were discussed and adaptations to the translation were only made if the wording was found to be meaningfully different from the original. For Bahasa Indonesia, no back-translation was done. In Israel, a dual-focus approach was used in which individuals from each of the linguistic groups develop the wording of the instructions and questions simultaneously in order to facilitate equality in clarity rather than linguistic equivalence (Peña, 2007).

Maternal beliefs about sensitive parenting. Maternal views of the ideal sensitive mother were assessed using the Maternal Behavior Q-Sort (MBQS; Pederson \& Moran, 1995; Pederson, Moran, \& Bento, 1999). The MBQS consists of 90 cards with statements about maternal behaviors that the mothers sorted into 9 stacks from "least descriptive" (1) to "most descriptive" (9) of the ideal mother. Because the original items were designed to be evaluated by professionals rather than mothers, the behavioral descriptions were simplified for the present study to make them more understandable for (low educated) mothers. For example, the item "Provides baby with little opportunity to contribute to the interaction" was simplified into "Gives her child little opportunity to play along or to respond" (see Appendix A for the full list of reworded items). The mothers were first asked to sort the cards into three stacks from "do not fit the ideal mother at all" to "fit the ideal mother really well." The mothers were explicitly told that there are no correct or wrong answers and that it is not about their own parenting behavior, but about what the ideal mother should or should not do. Any question they had concerning the meaning of an item was answered according to the item explanations in the protocol. When the mothers distributed the cards across the three stacks, they were asked to sort each stack into 3 smaller stacks. After the mothers distributed all cards across nine stacks, they were asked to evenly distribute the cards across the stacks until each stack consisted of 10 cards.

Consistent with standard Q-sort methodology, each mother's sort is represented as an individual variable for data analysis. This variable consists of 90 cases, representing the 90 cards, with scores from 1 to 9 reflecting the stack on which the mother put the card. A mother's sensitivity belief score was then computed by correlating her sort with the criterion sort. The criterion sort is provided by the authors of the MBQS (Pederson et al., 1999), and reflects the highly sensitive mother. Thus, a higher correlation refers to a greater overlap between the mother's beliefs about the ideal mother and attachment theory's notion of the highly sensitive mother.

Socio-demographic variables. Maternal educational level was measured on a 5-point scale: (1) primary school, (2) vocational school, (3) secondary school/middle vocational education, (4) high vocational education, and (5) and university or higher. Some minor adjustments were made to this classification system depending on the local context. Annual gross family income was measured on a 7-point scale that was defined differently in each country based on the national income distributions. In all countries score (1) referred to "no income" and score (7) referred to an income level considered to be very high (and above) in the country of interest. Mothers in Israel were not asked about their income level because this question would be perceived as violating privacy in the Israeli context. Mothers also reported on their age and their number of children. Further, each group was classified as urban versus rural by the research teams in each country, based on population density (much lower for rural than urban) and land use (mostly agricultural or fishing for rural). 


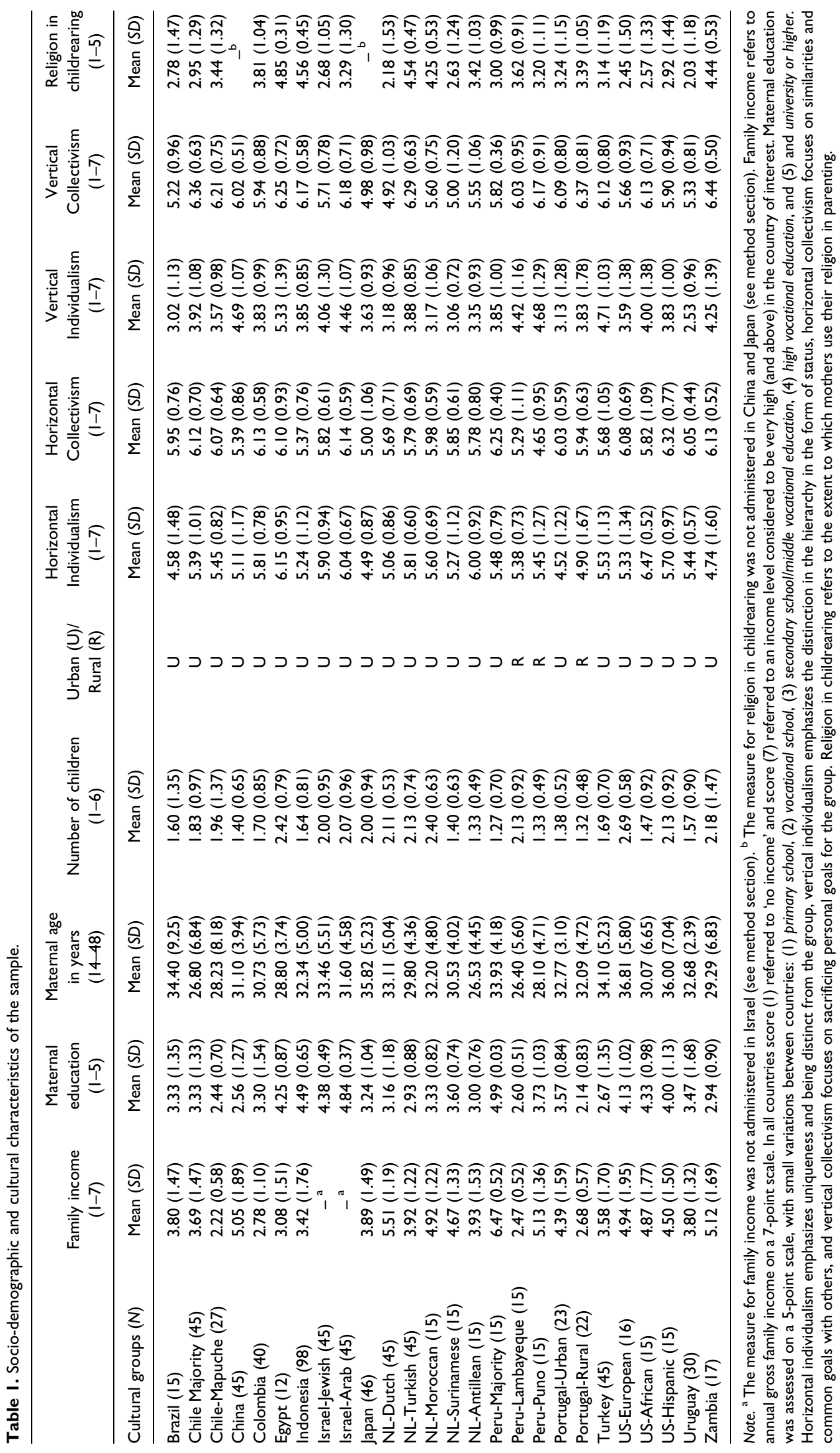


Cultural orientation. Mothers' cultural orientation was assessed with a 16-item short version of the Cultural Value Scale (Singelis et al., 1995; Triandis \& Gelfand, 1998), which is a questionnaire reflecting the dimensions collectivism versus individualism, and horizontal versus vertical relations. These dimensions form four scales, each emphasizing a specific cultural orientation (four items each): horizontal individualism emphasizes uniqueness and being distinct from the group, vertical individualism emphasizes the distinction in the hierarchy in the form of status, horizontal collectivism focuses on similarities and common goals with others, and vertical collectivism focuses on sacrificing personal goals for the group. The items were rated on a 7-point Likert scale ranging from strongly disagree (1) to strongly agree (7). Scale scores reflect item averages. Considering the limited number of items, the internal reliabilities of the four subscales were satisfactory (Cronbach's alphas .68 to .70). Descriptive statistics of the four cultural orientation scales are presented in Table 1.

Religion in parenting. Mothers also filled in a questionnaire (developed for this study) on the extent to which they use their religion in parenting. This questionnaire consisted of four items rated on a 5-point Likert scale ranging from (1) totally disagree to (5) totally agree. The items were "I use my religion as a guideline for the parenting of my child," "My religion helps me to raise my child well," "I teach my child a lot about my religion," and "I teach my child that religion plays an important role in our lives." An average item score was computed for the analyses. When mothers indicated not having a religion, the final score was set at zero. This questionnaire was not included in China and Japan, as the questions were deemed to be inappropriate to the cultural contexts of these countries. The internal consistency of the scale was high (Cronbach's alpha = $.95)$. Descriptive statistics of the religion in parenting scale are presented in Table 1.

\section{Data analysis}

Data inspection and analyses to test the hypotheses of the study were conducted with IMB SPSS Statistics, version 21.0 for Windows (SPSS Inc., Chicago, IL). Data inspection was performed within each of the 26 cultural groups. Outliers $(z>|3.29|, p=.001$, cf. Tabachnick \& Fidell, 2007) were found for MBQ sensitivity belief score (in total, 6 cases from 5 groups), family size ( 7 cases from 5 groups), horizontal individualism ( 2 cases from 2 groups), horizontal collectivism ( 2 cases from 2 groups), vertical collectivism ( 2 cases from 2 groups), and religion in parenting (1 case). These values were winsorized to bring them closer to the rest of the distribution within the relevant groups (Tabachnick \& Fidell, 2007).

Missing values were present on most of the predictor variables, including income (14\% missing values), number of children $(<1 \%$ missing values), maternal age ( $6 \%$ missing values), the four cultural orientation scales (1\% missing values on each), and religion in parenting (13\% missing values). Little's (1988) MCAR (missing completely at random) test revealed that the assumption of missing completely at random (Little \& Rubin, 2002) was violated, $\chi^{2}(111)$ $=338.84, p<0.01$. For variables with more than $5 \%$ of cases with missing values, $t$ tests were conducted to examine whether participants with missing values differed from those without missing values on other relevant variables. Income was missing in $14 \%$ of the cases, which was almost entirely due to the fact that income data was not collected in the two Israel samples (Jewish and Arab), which accounts for 90 out of 107 missing cases on this variable. Results of the $t$ tests comparing cases with missing values on income to other cases therefore represent differences between the Israel samples and all other samples that can also be derived from Table 1 (i.e., higher education, more children, higher horizontal individualism and collectivism). Religion in childrearing was missing in $13 \%$ of cases, which was almost entirely due to the fact that these questions were not asked in China and Japan (see Methods section), which accounts for 91 of 99 missing cases on this variable. Results of the $t$ tests comparing cases with missing values on religion in childrearing to other cases therefore represent differences between the Japanese and Chinese samples and all other samples that can also be derived from Table 1 (i.e., higher maternal age, lower education due to stratified sampling, lower horizontal individualism and collectivism). Finally, maternal age was missing in $6 \%$ of the cases (the majority of which were from Indonesia, Uruguay, rural Peru and China), and $t$ tests revealed that participants with missing values on age had significantly fewer children than other participants.

Because of the violation of MCAR assumption, data were substituted using multiple imputation under a multilevel model (Rubin, 1987; Van Buuren, 2011). Whereas listwise deletion will only give unbiased results in statistical analysis when the data are missing completely at random (MCAR; Little \& Rubin, 2002), multiple imputation will also give unbiased results under the less strict assumption of missing at random (MAR; Little \& Rubin, 2002; Rubin, 1976). Although it cannot be tested whether the MAR assumption holds (Schafer, 1997, p. 22), multiple imputation will still give less biased results than listwise deletion (Schafer, 1997, p. 26) when this assumption is violated. It was therefore decided to use multiple imputation for handling the missing data rather than listwise deletion. Multiple imputation was carried out using the procedure mice.impute.2 1.norm in R (Van Buuren \& GroothuisOudshoorn, 2011). In total, 100 imputed data sets were generated, and the multilevel regression coefficients and their standard errors were pooled in SPSS, using Rubin's combination rules (1987). $-2 \log$ likelihoods and likelihood ratio tests were averaged across the multiply imputed data sets as a rough indication for the specific model's fit.

The first hypothesis regarding high convergence between mothers' view of the ideal mother and attachment theory's notion of sensitive parenting was tested in the following analyses. First, we computed correlation coefficients for each mother's MBQS sort and the criterion sort that reflects the highly sensitive parent. In other words: the rankings of the 90 items by the 751 mothers are used as cases (rather than variables), so they can be correlated with the criterion sort. High correlations of the mothers' sorts with the criterion sort would reflect high convergence between mothers' notion of the ideal mother and attachment theory's notion of the highly sensitive mother. Second, to further qualify high convergence, we then compared the average and range of correlations between maternal beliefs about the ideal mother and attachment theory's notion of the highly sensitive mother (MBQS findings in the current study) to the average and range found by Posada et al. (1995) with respect to maternal beliefs about the ideal child and attachment theory's notion of the securely attached child, which was reported to be high. We computed the $84 \%$ confidence intervals (Goldstein \& Healy, 1995; Julious, 2004) for our MBQS findings and for Posada et al.'s AQS findings. If the $84 \%$ confidence 
International Journal of Behavioral Development

intervals overlap, this means that the two distributions may not be significantly different from each other.

The second set of hypotheses regarding socio-demographic and cultural predictors of convergence between maternal beliefs about the ideal mother and attachment theory's notion of the highly sensitive mother was tested with multilevel analyses. With these analyses, we examined the role of shared cultural group membership in variations of mother-criterion convergence (i.e., the correlation coefficients between each mother's sort and the criterion sort), after taking into account socio-demographic variables (i.e., rural versus urban, family income, maternal education, maternal age, and family size). We did not include child age as a predictor in the multilevel model, as most mothers had more than one child, and many also had two children within the target age range. Child age (of any of the children in the family) was not significantly related to sensitivity beliefs, with $p$ values ranging from .13 to .95 . Furthermore, we tested whether cultural factors such as cultural orientation and religiosity in parenting explained some of the variance left after co-varying the socio-demographic variables. We did not include type of religion as a predictor, because a categorical variable is difficult to use in multilevel analyses. Further, type of religion was strongly confounded with cultural group membership (e.g., all Portuguese mothers self-identified as Catholic, all Egyptians as Muslim, etc.), and the rural/urban distinction (all rural mothers were Catholic). Each of the predictors was centered. The multilevel model included a random intercept, allowing for mean differences in MBQS scores between the 26 different cultural groupings. In the modeling process, random effects for each of the centered sociodemographic and attitude variables were added to the null model (Twisk, 2011), and none of these random effects were significant. Consequently, the predictor variables were added as fixed effect only covariates in two blocks in two subsequent models, and for each step model fit was evaluated by the change in -2 log likelihood (tested as $\chi^{2}$, with $d f$ equal to the number of parameters added) for each step in the model. The $-2 \log$ likelihood was presented in smaller-is-better form. See Appendix B for the formulas for the final model.

\section{Results}

Table 2 shows the average agreement of mothers in each cultural group with the criterion sort for the MBQS (see data analysis section for details on calculation). In support of our first hypothesis, the overall average correlation coefficient was .68 , indicating a strong convergence between maternal ideas about the ideal mother and attachment theory's notion of the highly sensitive mother. Further, we found that agreement within groups, with an average of .52 (range .38-.62), was similar to agreement between groups, with an average of .44 (range .43-.58). Comparing the $84 \%$ confidence intervals for within group agreement (.65-.71) and between-group agreement (.63-.66) showed that these overlapped, indicating that they were not significantly different from each other. Table 3 shows the items that were evaluated as most and least like the ideal mother across the 26 cultural groups. It shows that the majority of top items refer to different aspects of sensitivity, including proximity/interaction, signal perception, and appropriate positive responsiveness. The bottom items reflect lack of responsiveness, and negative or flat affect. We further compared the average and range of correlations between maternal beliefs about the ideal mother and attachment theory's notion of the highly sensitive mother $(M=.68$;
Table 2. Correlations with criterion sort reflecting the highly sensitive mother.

\begin{tabular}{|c|c|c|c|c|c|}
\hline $\begin{array}{l}\text { Cultural } \\
\text { group }(N)\end{array}$ & M & (Range) & Cultural group $(N)$ & $M$ & (Range) \\
\hline Brazil (I5) & .70 & $(.55-.83)$ & NL-Surinamese (I5) & .76 & $(.69-.83)$ \\
\hline Chile-Majority (45) & .70 & $(.54-.83)$ & NL-Antillean (15) & .71 & $(.63-78)$ \\
\hline Chile-Mapuche (27) & .68 & $(.14-.84)^{\mathrm{a}}$ & Peru-Lima (I5) & .72 & $(.52-.82)$ \\
\hline China (45) & .70 & $(.37-.84)^{\mathrm{a}}$ & Peru-Lambayeque (I5) & .46 & $(.05-.78)$ \\
\hline Colombia (40) & .71 & $(.5 \mathrm{I}-.86)$ & Peru-Puno (15) & .47 & $(-.47-.73)$ \\
\hline Egypt (12) & .61 & $(.48-.82)$ & Portugal-Urban (23) & .67 & $(.51-.79)$ \\
\hline Indonesia (98) & .62 & $(.23-.78)^{\mathrm{a}}$ & Portugal-Rural (22) & .57 & $(.22-.77)$ \\
\hline Israel-Jewish (45) & .72 & $(.42-.85)$ & Turkey (45) & .68 & $(.38-.82)$ \\
\hline Israel-Arab (45) & .69 & $(.28-.81)^{\mathrm{a}}$ & US-European (16) & .71 & $(.57-.85)$ \\
\hline Japan (46) & .70 & $(.50-.83)$ & US-African (I5) & .64 & $(.24-.80)$ \\
\hline NL-Dutch (45) & .74 & $(.67-.85)$ & US-Hispanic (I5) & .71 & $(.47-.82)$ \\
\hline NL-Turkish (15) & .71 & $(.5 \mathrm{I}-.82)$ & Uruguay $(30)$ & .66 & $(.15-.81)^{\mathrm{a}}$ \\
\hline NL-Moroccan (15) & .70 & $(.41-.80)$ & Zambia (17) & .64 & $(.25-.79)$ \\
\hline
\end{tabular}

Note. ${ }^{\text {a }}$ These ranges and averages include non-winsorized outliers (all on the low side of the distribution). All further analyses were conducted with the winsorized data.

range $=.46-.76)$ to the average and range found by Posada et al. (1995) with respect to maternal beliefs about the ideal child and attachment theory's notion of the securely attached child $(M=.58$; range $=.47-.67)$. Results showed that the $84 \%$ confidence intervals for our MBQS findings (.66-.72) and for Posada et al.'s AQS findings (.53-.63) did not overlap, indicating that the mother-criterion agreement on the MBQS is significantly higher than the mother-criterion agreement on the AQS.

Our second and third sets of hypotheses were tested using multilevel analyses (see Table 4). Testing the null model, we found that the MBQS score depended on cultural group membership. In this model, mothers from the same cultural group had more similar ideas about the ideal mother compared to mothers from another cultural group, $\mathrm{ICC}=.297(p<.001)$. The proportion of explained variance $($ ICC $\times 100)$ was $29.7 \%$. Adding ethnic minority status (e.g., the migrant or indigenous groups in some countries) did not result in a significant random effect of minority status or a significant change in ICC of cultural group. When we included minority status as the sole random effect, the ICC amounted to a non-significant .008 (i.e., less than $1 \%$ explained variance in sensitivity beliefs). Minority status was thus not included in further analyses.

Inclusion of socio-demographic variables resulted in a significant change in the fit parameter $-2 \log$ likelihood compared to the random effect only model: mean $\chi^{2}(d f=5)=76.73$ (averaged across imputed data sets), $p<.01$. The variance explained by cultural group membership decreased from $29.7 \%$ to $10.0 \%$. Adding the cultural variables in the next step did not significantly improve the fit of the model, mean $\chi^{2}(d f=5)=10.90, p>.05$, although it showed a trend in that direction. The variance explained by cultural group membership decreased only marginally from $10.0 \%$ to $9.0 \%$. Significant predictors in the final equation were family income, family size, rural versus urban residence, and horizontal collectivistic values (see Table 4). Without the cultural groups for whom some variables had to be estimated (i.e., for the two groups in Israel: family income, for China and Japan: religion in parenting), the multilevel results remained basically the same, with the same significant model changes and predictors.

To explore whether the mothers from the three rural areas (two Peruvian indigenous groups, and one Portuguese rural group) 
Table 3. The 10 highest-scoring and 10 lowest-scoring maternal behavior Q-Sort items across all 26 cultural groups $(N=751)$.

\begin{tabular}{|c|c|c|}
\hline $\begin{array}{l}10 \text { Highest-scoring } \\
\text { items }\end{array}$ & $\begin{array}{l}\text { Mean item } \\
\text { score }\end{array}$ & $\begin{array}{l}\text { Number of cultural groups in } \\
\text { which the item is in Top } 20\end{array}$ \\
\hline $\begin{array}{l}\text { I. Shows affection by } \\
\text { touching (Affect) }\end{array}$ & 8.16 & 25 \\
\hline $\begin{array}{l}\text { 2. Show child she is happy } \\
\text { with him/her (Affect) }\end{array}$ & 7.84 & 24 \\
\hline 3. Praises child (Sensitivity) & 7.62 & 22 \\
\hline $\begin{array}{l}\text { 4. Shows enjoyment of } \\
\text { child (Affect) }\end{array}$ & 7.62 & 19 \\
\hline $\begin{array}{l}\text { 5. Encourages trying new } \\
\text { things (Sensitivity) }\end{array}$ & 7.61 & 24 \\
\hline $\begin{array}{l}\text { 6. Seeks contact with child } \\
\text { (Sensitivity) }\end{array}$ & 7.61 & 26 \\
\hline $\begin{array}{l}\text { 7. Is cheerful with child } \\
\text { (Affect) }\end{array}$ & 7.60 & 20 \\
\hline $\begin{array}{l}\text { 8. Interrupts dangerous } \\
\text { activities (Sensitivity) }\end{array}$ & 7.58 & 24 \\
\hline $\begin{array}{l}\text { 9. Talks to child } \\
\text { (Sensitivity) }\end{array}$ & 7.56 & 21 \\
\hline $\begin{array}{l}\text { 10. Responds well when } \\
\text { child is sad (Sensitivity) }\end{array}$ & 7.54 & 21 \\
\hline 10 Lowest-scoring items & $\begin{array}{l}\text { Mean item } \\
\text { score }\end{array}$ & $\begin{array}{c}\text { Number of cultural groups in } \\
\text { which the item is in } \\
\text { Bottom } 20\end{array}$ \\
\hline 90. Negative, hostile & 1.35 & 26 \\
\hline 89. Never responds & 1.46 & 26 \\
\hline 88. Treats child as object & 1.64 & 26 \\
\hline 87. Insincere affection & 1.94 & 24 \\
\hline $\begin{array}{l}\text { 86. Irritated if child wants } \\
\text { to sit on her lap }\end{array}$ & 2.09 & 25 \\
\hline $\begin{array}{l}\text { 85. Does not respond to } \\
\text { sounds, smiles }\end{array}$ & 2.13 & 24 \\
\hline $\begin{array}{l}\text { 84. Not at ease holding } \\
\text { child }\end{array}$ & 2.17 & 23 \\
\hline 83. Aloof & 2.20 & 21 \\
\hline 82. Scolds, criticizes & 2.25 & 23 \\
\hline $\begin{array}{l}\text { 81. Responds only to } \\
\text { prolonged distress }\end{array}$ & 2.48 & 21 \\
\hline
\end{tabular}

Note. Item scores can range from I to 9 .

formed a homogenous subgroup, we calculated their intergroup agreement by correlating each mothers' sort from each group with all sorts from each of the other groups. The results showed agreement ranging from .34 to .41 , which is much lower than the agreement between urban groups, which ranged from .56 to .81 .

\section{Discussion}

In this study of 751 mothers from 26 cultural groups from 15 countries around the globe, a pattern of strong convergence between maternal beliefs about the ideal mother and attachment theory's descriptions of the highly sensitive mother across cultural groups was found. Further, individual variation in this convergence was significantly predicted by cultural group membership, rural versus urban residence, family income, family size, and horizontal collectivism. Our findings confirm the hypothesis that across cultures maternal beliefs about the ideal mother overlap considerably with the notion of sensitive responsiveness, a key construct in attachment theory. This result adds to the growing body of literature that points towards the universal applicability of the basic tenets of attachment theory.

Looking at the items that were considered to be highly ideal by all cultural groups, we noted that they include core sensitivity items related to signal perception and interpretation that received high scores by attachment theory experts describing a sensitive parent. The top items across cultural groups also include items referring to positive affect and warmth. Although positive affect is not synonymous with sensitivity and can co-occur with low responsiveness (Mesman \& Emmen, 2013), the combination of high positive affect and high responsiveness is characteristic of the sensitive mother and as such are also ranked high in the MBQs criterion sort. Interestingly, the top-scoring items across cultures also included items referring to close physical contact (such as "displays affection by touching") usually thought to be far more common as a part of sensitive parenting in non-Western cultures emphasizing relatedness than in Western cultures emphasizing autonomy (e.g., Keller et al., 2006).

Interestingly, the average and range of correlations between maternal beliefs about the ideal mother and attachment theory's notion of the highly sensitive mother were higher than the average and range found by Posada et al. (1995) with respect to maternal beliefs about the ideal child and attachment theory's notion of the securely attached child. Apparently, the cross-cultural agreement on maternal aspects of attachment (i.e., the importance of sensitive parenting) is stronger than cross-cultural agreement on child aspects of attachment (i.e., the importance of child secure-base behavior). This may be partly due to the salience of child age in early childhood. Given the large developmental differences between children of varying ages within the period spanning infancy, toddlerhood, and preschool, the (ideal) behaviors of young children in terms of seeking contact with caregivers and exploring the environment (the major elements of the secure-base concept and the AQS) may be influenced by the ages of a mother's own children. A mother of a 1-year-old may then characterize the ideal child differently than a mother who (also) has a 4-year-old child, as they are likely to base their notion of the ideal child at least partly on their experiences with their own children. Conversely, the notion of sensitive parenting is far less dependent on child age, as responding to children's signals contingently and appropriately is essentially relevant to all ages.

In a related vein, and in keeping with the organizational approach to the attachment relationship (Sroufe \& Waters, 1977), the items of the MBQS are often formulated in a somewhat abstract way leaving room for variations in behavioral manifestations (e.g., "her behavior fits the mood of her child"). The MBQS' focus on the function of parental behavior (responsiveness in a way that satisfies the child and provides a predictable and safe environment) rather than on its particular behavioral manifestations fits with the original conceptualization of the sensitivity construct (Ainsworth et al., 1974). As such, using more abstract formulations does justice to the breadth of behaviors that sensitivity may encompass, and reflects an organizational approach to the attachment relationship in that neither parent nor child behaviors can be captured by a predefined and exhaustive set of concrete behaviors (Sroufe \& Waters, 1977). This approach also allows for both convergence and differences between cultures and does not necessitate the adoption of an "either-or" perspective. From an attachment theory perspective, it does not matter what a mother does to soothe her crying infant (extreme behaviors excluded), as long as the infant is soothed. In 
Table 4. Socio-demographic and cultural predictors of maternal behavior q-sort sensitivity belief scores $(N=75 \mathrm{I})$.

\begin{tabular}{|c|c|c|c|c|c|c|}
\hline & \multicolumn{2}{|r|}{ Model 0} & \multicolumn{2}{|r|}{ Model I } & \multicolumn{2}{|r|}{ Model 2} \\
\hline & B & (95\% Confidence interval) & B & (95\% Confidence interval) & B & ( $95 \%$ Confidence interval) \\
\hline \multicolumn{7}{|l|}{ Fixed effects } \\
\hline Intercept & & & 0.822 & $(0.747-0.898)^{* *}$ & 0.741 & $(0.635-0.848)^{* * *}$ \\
\hline Family income & & & 0.006 & $(0.001-0.012)^{*}$ & 0.006 & $(0.001-0.012)^{*}$ \\
\hline Maternal education & & & 0.009 & $(0.00 \mathrm{I}-0.017)^{*}$ & 0.007 & $(-0.001-0.016)$ \\
\hline Maternal age & & & 0.001 & $(0.001-0.017)$ & 0.001 & $(-0.00 \mathrm{I}-0.002)$ \\
\hline Number of children & & & -0.017 & $(-0.027--0.008)^{* *}$ & -0.018 & $(-0.027--0.008)^{* *}$ \\
\hline Rural versus urban & & & -0.179 & $(-0.228--0.130)^{* *}$ & -0.174 & $(0.221-0.126)^{\text {*k }}$ \\
\hline $\begin{array}{l}\text { Horizontal } \\
\text { individualism }\end{array}$ & & & & & -0.002 & $(-0.010-0.005)$ \\
\hline Vertical individualism & & & & & -0.002 & $(-0.009--0.005)$ \\
\hline Horizontal collectivism & & & & & 0.013 & $(0.003-0.023)^{\text {*k }}$ \\
\hline Vertical collectivism & & & & & 0.005 & $(-0.005--0.015)$ \\
\hline Religion in childrearing & & & & & -0.002 & $(-0.008-0.004)$ \\
\hline \multicolumn{7}{|l|}{ Random effects } \\
\hline Intercept & 0.004 & $(0.002-0.007)^{* *}$ & 0.001 & $(0.000-0.002)^{* *}$ & 0.001 & $(0.000-0.002)^{*}$ \\
\hline Residual & 0.010 & $(0.009-0.011)^{* *}$ & 0.010 & $(0.009-0.011)^{* *}$ & 0.010 & $(0.000-0.011)^{* *}$ \\
\hline \multicolumn{7}{|l|}{ Model fit statistics } \\
\hline-2 log likelihood & -1235.16 & & -1311.89 & & -1322.79 & \\
\hline ICC Cultural group & $0.297^{* *}$ & & $0.101^{* *}$ & & $0.091 *$ & \\
\hline
\end{tabular}

Note. $*_{p}<.05 ; * * p<.01$.

other words, multiple behaviors can serve the same function (Bornstein, 2012; Sroufe \& Waters, 1977). Even though child behaviors within the attachment framework are also viewed from an organizational perspective, the ways in which a child can signal the need for parental proximity or attention are likely to be somewhat less numerous than the ways in which a parent can respond to such child signals.

Although the results of the current study point towards a universal appreciation of sensitive parenting, we did find a significant effect of cultural group membership on sensitivity beliefs. This effect became considerably smaller after taking into account socio-demographic predictors, but still remained significant. Interestingly, the set of cultural orientation measures in our study did not add significantly to the prediction of beliefs about the ideal mother as being a sensitive mother. Horizontal collectivism did emerge as a positive individual predictor of sensitivity beliefs, and reflects an emphasis on within-group identification and shared goals. Interestingly, a collectivistic cultural orientation focused on group harmony rather than individual autonomy has been suggested to be less conducive to sensitivity (e.g., Keller, 2013). However, combined with the horizontal idea of equality, it reflects the group as a social context without the automatic connotation of pressure on individuals to conform to a preset norm. Example items from the horizontal collectivism scale are "To me, pleasure is spending time with others," and "I feel good when I cooperate with others." Thus, it appears that individuals who see the social group as a positive collaborative social system are also more likely to endorse beliefs about parenting that relate to proximity and positive responsiveness to individual children. This is consistent with previous findings that a horizontal cultural orientation is related to authoritative parenting practices that relate to treating children as individuals to be respected (e.g., Chirkov et al., 2005), which is consistent with the notion of non-intrusive sensitive responsiveness.

The effect of cultural group membership on sensitivity beliefs above and beyond the socio-demographic and cultural predictors needs further investigation. In particular, the specific demands of the cultural and geographical context on family life may provide more insights into how parenting beliefs develop. The socioeconomic factors investigated in this study may be too broad to capture more subtle everyday life demands that vary across contexts and may differentially impact maternal beliefs. Further, the contextspecific nature and levels of demands on parents may also lead to varying levels of maternal stress. Consistent with the Family Stress Model, this might further explain cultural group differences in sensitivity beliefs. Future studies could also include more diverse measures of cultural orientation to find out which particular cultural values explain some of the cultural group effect. For instance, they could look at parental ethnotheories specifically related to autonomous or relatedness parenting goals that have been found to distinguish between cultural and geographic (urban/rural) groups in terms of parenting patterns in other studies (e.g., Keller et al., 2006). However, the way it was used in our study, the MBQS may in fact be considered a measure of ethnotheories about parenting which also showed the ability to distinguish between urban and rural mothers, and mothers from different cultural groups. In that sense, the current study confirms previous findings that parenting beliefs vary according to the specific developmental niche that provides the cultural context for childrearing practices (e.g., Bornstein et al., 2012; Harkness \& Super, 1992; Keller et al., 2006). It may be interesting for understanding culture-specific patterns of sensitivity to examine which concrete behaviors are seen as ideal to ensure a secure child in different cultures. There is indeed evidence that concrete responsive behaviors in mother-infant interactions differ between cultural groups (e.g., Kärtner, Keller, \& Yovsi, 2010; True et al., 2001). It is thus important to note that the universality hypothesis does leave room for regional variations in the manifestations of attachment-related behaviors depending on the developmental niche in which families function (Mesman et al., in press).

A strong predictor of a higher convergence between mothers' description of the ideal mother and the notion of the highly 
sensitive parent was urban versus rural residence, with higher sensitivity belief scores for mothers living in urban areas. This consistent with previous findings that level of urbanization is an important factor in distinguishing between different developmental niches (e.g., Keller et al., 2006). Rural parents have generally been found to endorse more authoritarian parenting styles and have less knowledge about child development than urban parents (e.g., Miller \& Votruba-Drzal, 2013; Nacak et al., 2011; Pinderhughes, Nix, Foster, \& Jones, 2007), which points towards a potentially lower emphasis on child-centered sensitive responsiveness. However, the agreement between the three rural groups (two Peruvian indigenous, and one Portuguese rural group) was also lower than agreement between the urban groups. Apparently, living in a rural area alone does not explain beliefs about the ideal mother, and other factors are at play. Maybe it is important to distinguish between mothers who come from families who have lived rurally for generations and those who come from families who moved there recently. Rural areas may also differ regarding the proximity and availability of resources relevant to family life such as schools, health care, and social networks. Such contextual variations might also relate to how mothers view the role of parents. The finding that the urban groups did show strong agreement on the characteristics of the ideal mother suggests that the globalized urban environment serves as an equalizer in terms of beliefs about parenting.

The two other socio-demographic predictors that remained significant in the final models were family income and family size, with mothers with lower family incomes and more children having lower sensitivity belief scores than mothers with a higher income and fewer children. The finding regarding family income is consistent with evidence from the study examining sensitivity beliefs in different cultural groups in the Netherlands (Emmen et al., 2012). That study did not find family size to be a significant predictor of sensitivity beliefs, but that may have been due to the rather restricted range in that sample (almost $90 \%$ had 2 or 3 children). The results regarding family income fits with the basic assumptions of the Family Stress Model (Conger \& Donellan, 207) that describes how economic pressures can lead to parental stress, which in turn predicts less optimal parenting behaviors. It has been suggested that highly stressed parents (due to economic pressures and/or large family size) are less likely to think of parenting as a significant emotional and time investment (i.e., sensitive parenting), because they are more concerned with day-to-day problems, which may lead to the experience of parenting as mainly aiming to control child behavior (McLoyd, 1998; Pinderhughes et al., 2000).

An important strength of the current study is the inclusion of multiple non-Western samples from countries and cultural groups that are rarely represented in research on parenting and child development. Indeed, the vast majority of studies in our field rely on WEIRD samples, that is, those recruited from Western Educated, Industrialized, Rich, and Democratic countries (Henrich, Heine, $\&$ Norenzayan, 2010). The current study included 11 countries that are not WEIRD, and also included indigenous populations from South-American countries that are rarely studied in parenting research. Another strength is the inclusion of a variety of sociodemographic and cultural predictors that allowed us to provide a more layered interpretation of the effect of culture on maternal sensitivity beliefs, and to show how both culture and sociodemographic characteristics contribute to parenting beliefs. Finally, beliefs about sensitivity have not yet been examined before within or across cultures, and our study thus provides an important first step in understanding how group differences in sensitivity beliefs may underlie group differences in sensitive parenting behaviors. It is then crucial to further study the link between sensitivity beliefs and behaviors.

Some limitations of the current study need to be mentioned. First, the samples mostly represent convenience samples and the group sizes within each country and cultural group were generally small, in some cases very small, and only included a few small rural groups. This limits the generalizability of the findings to the countries, regions, and ethnic groups as a whole. However, the strength of the Q-sort method is that agreement between groups is calculated by correlating each sort with each of the other sorts in a way that capitalizes on the small sample size and on the large number of items (90). Nevertheless, future studies should aim to include more rural groups and larger samples. Limited representativeness of the samples was also due to the research methods that required some basic level of literacy. Similarly, we could not examine the role of type of religion, because this variable was highly confounded with cultural group membership and the rural/urban distinction. Future studies should aim to collect data from diverse religious groups within regions and countries to address this issue.

A second limitation is that the procedures for translating the original English (reworded) items into each of the nine languages represented in our study were not standardized. However, this does not appear to have affected our results in terms of agreement between mothers and the attachment theory criterion, because in cases where the same language was used in different subgroups (e.g., Portuguese in two Portuguese groups and Brazil, and Spanish in several South-American countries), the average agreement for these groups were not uniformly lower or higher than those found for other groups. Third, the scope of the study is limited by the inclusion of only mothers. Paternal or grandparental beliefs about parenting may differ from maternal beliefs and may provide a different cross-cultural picture. Further, in some cultural contexts, other caregivers such as older siblings and other family members may also be central to early childhood caregiving and should be included in future studies on caregivers' beliefs about parenting in different cultures. Finally, the socio-demographic predictors included in this study might not fully capture the cultural niches represented in our sample. However, it is difficult to quantitatively measure the rather abstract notion of a cultural niche and demographic, economic, and religious characteristics as well as cultural values may be the best available proxies.

In conclusion, the results of the current study add to the body of literature providing evidence for both culture-general and culturespecific characteristics of parenting. Across 26 cultural groups from across the globe, mothers' ideas about the ideal mother were found to overlap substantially with the notion of the highly sensitive mother, pointing towards a universal appreciation of the importance of contingent responsiveness in parenting young children. This finding suggests important opportunities for parenting interventions aimed at families with young children in multi-cultural settings. Even when parents come from very different cultures than professionals in family support services, they are likely to agree on the importance of sensitive parenting, which may open up valuable avenues for joint goals in parenting interventions. On the other hand, we also found a significant effect of cultural group on sensitivity beliefs that was largely, but not entirely, due to sociodemographic factors, and especially rural versus urban residence. 
These findings emphasize the importance of further investigating mechanisms that explain how culture relates to maternal beliefs about sensitive parenting, as well as the inclusion of rural groups in parenting research. Overall, the ideal mother is very similar to a sensitive mother across the globe, with regional and cultural variations in the strength of this pattern.

\section{Acknowledgements}

We would like to thank Kerri Chladnicek, Hannah Knafo and Adella Nikitiades for their contribution to data collection in the U.S., and Marjolein Branger for her support in organizing the data and the references.

\section{Funding}

This research received no specific grant from any funding agency in the public, commercial, or not-for-profit sectors.

\section{References}

Ainsworth, M. D. S. (1967). Infancy in Uganda: Infant care and the growth of love. Baltimore, MD: Johns Hopkins Press.

Ainsworth, M. D. S., Bell, S. M., \& Stayton, D. J. (1974). Infant-mother attachment and social development. In M. P. Richards (Ed.), The introduction of the child into a social world (pp. 99-135). London, UK: Cambridge University Press.

Bakermans-Kranenburg, M. J., Van IJzendoorn, M. H., \& Juffer, F. (2003). Less is more: Meta-analyses of sensitivity and attachment interventions in early childhood. Psychological Bulletin, 129, 195-215. doi:10.1037/0033-2909.129.2.195

Bornstein, M. H. (2012). Cultural approaches to parenting. Parenting: Science \& Practice, 12, 212-221. doi:10.1080/15295192.2012. 683359

Bornstein, M. H., Putnick, D. L., Suwalsky, J. T. D., Venuto, P., De Falco, S., Zingman de Galperin, C., ... Heslington Tichovolsky, M. (2012). Emotional relationships in mothers and infants: Culture-common and culture-specific characteristics of dyads from rural and metropolitan settings in Argentina, Italy, and the United States. Journal of Cross-Cultural Psychology, 43, 171-197. doi: 10.1177/0022022110388563

Bowlby, J. (1969). Attachment and loss (vol. 1). New York, NY: Basic Books.

Boyd, R., \& Richerson, P. J. (2005). The origin and evolution of cultures. New York, NY: Oxford University Press.

Chirkov, V. I., Ryan, R. M., \& Willness, C. (2005). Cultural context and psychological needs in Canada and Brazil: Testing a selfdetermination approach to the internalization of cultural practices, identity, and well-being. Journal of Cross-Cultural Psychology, 36, 423-443. doi:10.1177/0022022105275960

Clément, M.-È., \& Chamberland, C. (2009). The role of parental stress, mother's childhood abuse and perceived consequences of violence in predicting attitudes and attribution in favor of corporal punishment. Journal of Child and Family Studies, 18, 163-171. doi:10.1007/s10826-008-9216-z

Conger, R. D., \& Donnellan, M. B. (2007). An interactionist perspective on the socioeconomic context of human development. Annual Review of Psychology, 58, 175-199. doi:10.1146/annurev.psych. 58.110405 .085551

Ding, Y., Xu, X., Wang, Z., Li, H., \& Wang, W. (2012). Study of mother-infant attachment patterns and influence factors in Shanghai. Early Human Development, 88, 295-300. doi:10.1016/j.earlhumdev.2011.08.023
Emmen, R. A. G., Malda, M., Mesman, J., Ekmekci, H., \& Van IJzendoorn, M. H. (2012). Sensitive parenting as a cross-cultural ideal: Sensitivity beliefs of Dutch, Moroccan, and Turkish mothers in the Netherlands. Attachment and Human Development, 14, 601-619. doi:10.1080/14616734.2012.727258

Feldman, R., \& Masalha, S. (2010). Parent-child and triadic antecedents of children's social competence: Cultural specificity, shared process. Developmental Psychology, 46, 455-467. doi:10.1037/ a0017415

Furman, W., \& Lanthier, R. (2002). Parenting siblings. In M. H. Bornstein (Ed.), Handbook of parenting. Volume 1: Children and parenting (2nd ed., pp. 165-188). Mahwah, NJ: Lawrence Erlbaum Associates.

Georgiou, S. N., Fousiani, K., Michaelides, M., \& Stavrinides, P. (2013). Cultural value orientation an authoritarian parenting as parameters of bullying and victimization at school. International Journal of Psychology, 48, 69-78. doi:10.1080/00207594.2012. 754104

Gojman, S., Millán, S., Carlson, E., Sánchez, G., Rodarte, A., González, P., \& Hernández, G. (2012). Intergenerational relations of attachment: A research synthesis of urban/rural Mexican samples. Attachment \& Human Development, 14, 553-566. doi:10.1080/14616734. 2012.727255

Goldstein, H., \& Healy, M. J. R. (1995). The graphical presentation of a collection of means. Journal of the Royal Statistical Society, 158, 175-177. doi:10.2307/2983411

Harkness, S., \& Super, C. M. (1992). Parental ethnotheories in action. In I. Sigel, A. V. McGillicuddy-DeLisi \& J. Goodnow (Eds.), Parental belief systems: The psychological consequences for children (pp. 373-392). Hillsdale, NJ: Erlbaum.

Henrich, J., Heine, S. J., \& Norenzayan, A. (2010). The weirdest people in the world? Behavioral and Brain Sciences, 33, 61-135. doi:10. 1017/S0140525X0999152X

Jin, M. K., Jacobvitz, D., Hazen, N., \& Jung, S. H. (2012). Maternal sensitivity and infant attachment security in Korea: Cross-cultural validation of the Strange Situation. Attachment \& Human Development, 14, 33-44. doi:10.1080/14616734. 2012.636656

Julious, S. A. (2004). Using confidence intervals around individual means to assess statistical significance between two means. Pharmaceutical Statistics, 3, 217-222. DOI:10.1002/pst.126

Kagitcibasi, C. (2007). Family, self, and human development across cultures: Theory and applications (2nd ed.). Mahwah, NJ: Erlbaum.

Kärtner, J., Keller, H., \& Yovsi, R. D. (2010). Mother-infant interaction during the first 3 months: The emergence of culture-specific contingency patterns. Child Development, 81, 540-554. doi:10.1111/j. 1467-8624.2009.01414.x

Keller, H. (2013). Attachment and culture. Journal of Cross-Cultural Psychology, 44, 175-194. doi:10.1177/0022022112472253

Keller, H., Borke, J., Staufenbiel, T., Yovsi, R. D., Abels, M., Papaligoura, Z., ... Su, Y. J. (2009). Distal and proximal parenting as alternative parenting strategies during infants' early months of life: A cross-cultural study. International Journal of Behavioral Development, 33, 412-420. doi:10.1177/ 0165025409338441

Keller, H., Lamm, B., Abels, M., Yovsi, R., Borke, J., Jensen, H., .. . Chaudhary, N. (2006). Cultural models, socialization goals, and parenting ethnotheories: A multicultural analysis. Journal of Cross-Cultural Psychology, 37, 155-172. doi:10.1177/ 0022022105284494 
Little, R. J. A., \& Rubin, D. B. (2002). Statistical analysis with missing data (2nd ed.). New York, NY: Wiley.

Mahoney, A., Pargament, K. I., Tarakeshwar, N., \& Swank, A. B. (2001). Religion in the home in the 1980s and 1990s: A meta-analytic review and conceptual analysis of links between religion, marriage, and parenting. Journal of Family Psychology, 15, 559-596. doi:10.1037/0893-3200.15.4.559

McLoyd, V. C. (1998). Socioeconomic disadvantage and child development. American Psychologist, 53, 185-204. doi:10.1037/0003066X.53.2.185

Mesman, J., \& Emmen, R. A. G. (2013). Mary Ainsworth's legacy: A systematic review of observational instruments measuring parental sensitivity. Attachment and Human Development, 15, 485-506. doi: 10.1080/14616734.2013.820900

Mesman, J., Van IJzendoorn, M. H., \& Bakermans-Kranenburg, M. J. (2012). Unequal in opportunity, equal in process: Parental sensitivity promotes positive child development in ethnic minority families. Child Development Perspectives, 6, 239-250. doi:10.1111/j. 1750-8606.2011.00223.x

Mesman, J., Van IJzendoorn, M. H., \& Sagi-Schwartz, A. (in press). Cross-cultural patterns of attachment: Universal and contextual dimensions. In J. Cassidy \& P. R. Shaver (Eds.), Handbook of attachment: Theory, research, and clinical applications (3rd ed.). New York, NY: Guilford Press.

Miller, P., \& Votruba-Drzal, E. (2013). Early academic skills and childhood experiences across the urban-rural continuum. Early Childhood Research Quarterly, 28, 234-248. DOI:10.1016/j.ecresq. 2012.12.005

Nacak, M., Yagmurlu, B., Durgel, E., \& van de Vijver, F. (2011). Parenting in metropole and Anatolia samples: The role of residence and education in beliefs and behaviors. Turkish Journal of Psychology, $26,85-100$.

Oyserman, D., Coon, H. M., \& Kemmelmeier, M. (2002). Rethinking individualism and collectivism: Evaluation of theoretical assumptions and meta-analyses. Psychological Bulletin, 128, 3-72. doi: 10.1037//0033-2909.128.1.3

Pederson, D. R., \& Moran, G. (1995). A categorical description of infant-mother relationships in the home and its relation to Q-sort measures of infant-mother interaction. Monographs of the Society for Research in Child Development, 60(2-3), 111-132. doi:10. 1111/j.1540-5834.1995.tb00207.x

Pederson, D. R., Moran, G., \& Bento, S. (1999). Manual Maternal Behavior Q-Sort Version 3.1. Retrieved from http://ir.lib.uwo.ca/ psychologypub/?utm_source=ir.lib.uwo.ca\%2Fpsychologypub $\%$ 2F1\&utm_medium $=$ PDF\&utm_campaign $=$ PDFCoverPages

Peña, E. D. (2007). Lost in translation: Methodological considerations in cross-cultural research. Child Development, 78, 1255-1264. doi: 10.1111/j.1467-8624.2007.01064.x

Pinderhughes, E. E., Dodge, K. A., Bates, J. E., Pettit, G. S., \& Zelli, A. (2000). Discipline responses: Influences of parents' socioeconomic status, ethnicity, beliefs about parenting, stress, and cognitiveemotional processes. Journal of Family Psychology, 14, 380-400. doi:10.1037/0893-3200.14.3.380

Pinderhughes, E. E., Nix, R., Foster, E. M., \& Jones, D. (2007). Parenting in context: Impact of neighborhood poverty, residential stability, public services, social networks, and danger on parental behaviors. Journal of Marriage and the Family, 63, 941-953. doi:10.1111/j. 1741-3737.2001.00941.x

Posada, G., Gao, Y., Wu, F., Posada, R., Tascon, M., Schöelmerich, A., ... Synnevaag, B. (1995). The secure-base phenomenon across cultures: Children's behavior, mother's preferences, and experts' concepts. Monographs of the Society for Research in Child Development, 60(2-3), 27-48. doi:10.1111/j.1540-5834. 1995.tb00202.x

Posada, G., Lu, T., Trumbell, J., Kaloustian, G., Trudel, M., Plata, S., \& Lay, K. L. (2013). Is the secure base phenomenon evident here, there, and anywhere? A cross-cultural study of child behavior and experts' definitions. Child Development, 84, 1896-1905.

Rothbaum, F., Weisz, J., Pott, M., Miyake, K., \& Morelli, G. (2000). Attachment and culture: Security in the United States and Japan. American Psychologist, 55, 1093-1104. doi:10.1037/0003-066X. 55.10 .1093

Rubin, D. B. (1976). Inference and missing data. Biometrika, 63, 581-592.

Rubin, D. B. (1987). Multiple imputation for nonresponse in surveys. New York, NY: Wiley.

Schafer, J. L. (1997). Analysis of incomplete multivariate data. London, UK: Chapman \& Hall.

Schlomer, G. L., \& Belsky, J. (2012). Maternal age, investment, and parent-child conflict: A mediational test of the terminal investment hypothesis. Journal of Family Psychology, 26, 443-452. doi:10. 1037/a0027859

Singelis, T. M., Triandis, H. C., Bhawuk, D., \& Gelfand, M. J. (1995). Horizontal and vertical dimensions of individualism and collectivism: A theoretical and measurement refinement. Cross-Cultural Research, 29, 240-275. doi:10.1177/106939719502900302

Sroufe, L. A., \& Waters, E. (1977). Attachment as an organizational construct. Child Development, 48, 1184-1199. doi:10.2307/ 1128475

Sternberg, K. J., \& Lamb, M. E. (1992). Evaluations of attachment relationships by Jewish Israeli day-care providers. Journal of CrossCultural Psychology, 23, 285-299.

Tabachnick, B. G., \& Fidell, L. S. (2007). Using multivariate statistics (5th ed.). New York, NY: Harper Collins.

Tomlinson, M., Cooper, P., \& Murray, L. (2005). The mother-infant relationship and infant attachment in a South African peri-urban settlement. Child Development, 76, 1044-1054. doi:10.1111/j. 1467-8624.2005.00896.x

Triandis, H. C., \& Gelfand, M. J. (1998). Converging measurement of horizontal and vertical individualism and collectivism. Journal of Personality and Social Psychology, 74, 118-128. doi:10.1037/ 0022-3514.74.1.118

True, M. M., Pisani, L., \& Oumar, F. (2001). Infant-mother attachment among the Dogon of Mali. Child Development, 72, 1451-1466. doi:10.1111/1467-8624.00359

Twisk, J. W. R. (2011). Applied multilevel analysis: A practical guide for medical researchers. New York, NY: Cambridge University Press.

Van Buuren, S. (2011). Multiple imputation of multilevel data. In J. J. Hox \& J. K. Roberts (Eds.), The handbook of advanced multilevel analysis (pp. 173-196). Milton Park, UK: Routledge.

Van Buuren, S., \& Groothuis-Oudshoorn, K. (2011). mice: Multivariate Imputation by Chained Equations in R. Journal of Statistical Software, 45, 1-67. doi:10.1.1.169.5745

Vereijken, C., Riksen-Walraven, J. M., \& Kondo-Ikemura, K. (1997). Maternal sensitivity and infant attachment security in Japan: A longitudinal study. International Journal of Behavioral Development, 21, 35-49. doi:10.1080/016502597384974

Vermeer, P. (2011). The impact of parental religiosity on parenting goals and parenting style: A Dutch perspective. Journal of Beliefs \& Values: Studies in Religion \& Education, 32, 69-84. doi:10. 1080/13617672.2011.549311 


\section{Appendix A}

Because the original items were designed to be evaluated by professionals rather than mothers, the behavioral descriptions were simplified for the present study to make them more understandable for (low-educated) mothers. The full list of simplified descriptions is provided below.

1. Gives her child little opportunity to play along or to respond.

2. Pays attention to what her child is doing when there is a visitor.

3. Her responses to her child are unpredictable.

4. Does not pay attention to her child when she is busy with a visitor.

5. Is not at ease when she is holding her child close (for instance on her lap).

6. Supports contact of her child with a visitor.

7. Treats her child as an object when holding him/her.

8. Lets her child know when she leaves the room.

9. Does not respond when her child makes sounds, smiles or reaches.

10. Speaks to her child directly and not just about her child.

11. Speaks slowly and repeats the words if she talks to her child.

12. Mother determines when her child has to sleep, whether her child is tired or not.

13. Uses brothers/sisters or television to keep her child entertained.

14. Suddenly stops playing with her child to talk to a visitor.

15. Tries to involve her child in games or activities that are actually too difficult for her child, but does not notice that.

16. Does not realize it when things become too much for her child.

17. Dictates what happens and how fast things go, not her child.

18. The house does not look like a child is living there.

19. Places her child in another room when her child is in a bad mood or cranky.

20. Responds well when her child is sad.

21. Finds it difficult to take care of her child.

22. Seems to be unaware when her child is asking for attention.

23. Makes sure that her child can always come closer to her.

24. Makes sure her child can hear or see her.

25. Is not very good at dividing her attention between her child and other tasks, so that she does not always see what her child needs.

26. Responds immediately when her child cries/whimpers.

27. Responds when her child asks for attention, even when she is busy with a visitor.

28. Offers her child something else to do to distract him/her from something that is not allowed.

29. When her child is distressed, mother understands why.

30. Uses mainly physical contact with her child instead of using her voice.

31. Distracts her child to something else when her child wants to sit on her lap, without a gentle transition.

32. Mother does not follow her child with her behaviors.

33. Tries several different things to satisfy her child, without a clear plan.

34. Her behavior fits the mood of her child.

35. Finishes activities and games with her child properly so that her child is content.

36. Steps in when her child does something dangerous.

37. Steps in when her child does something that can make him/her dirty.
38. Provides healthy snacks.

39. Tries to teach her child things during play.

40. Encourages her child to feed him-/herself if her child wants to.

41. Her contact with her child consists mostly of doing things (e.g., eating, or playing with toys).

42. Her way of showing affection for her child seems insincere.

43. Is cheerful when she does things with her child.

44. Knows what her child can and can not do at his/her age when it comes to self-control.

45. Praises her child/gives her child compliments.

46. Makes sure her child is comfortable on her lap.

47. Shows her affection for her child by touching her child or cuddling him/her.

48. Points to interesting things in her child's environment and tells him/her what they are called.

49. Seeks contact with her child.

50. Makes sure that the environment is interesting for her child.

51. Makes sure that there are toys that fit the age of her child.

52. If she wants to forbid her child something, she does so with words and without touching or restraining the child.

53. Waits for her child's response when they are doing something together.

54. Teases her child to keep her child's attention, even when the child does not like it.

55. Sees her child as a person with his/her own wishes and even accepts it when her child wants to do things that she does not like.

56. Has fixed ideas about how her child needs to be taken care of and always does these things the same way.

57. Shows that she enjoys doing things with her child.

58. Takes her child's needs into account in the way the house is furnished/organized.

59. Lets her child do things he/she likes without interruption.

60. Often scolds or criticizes her child.

61. Is irritated when her child wants to sit on her lap.

62. Understands her child well as can be seen from the responses of her child.

63. Shows that she is aware of her child's distress but does not respond.

64. Greets her child when she comes back into the room.

65. Responds to what her child does or says.

66. Never responds to her child.

67. Responds only when her child shows prolonged or intense distress.

68. Adapts her tempo and tone to what her child wants when they are playing together.

69. Notices when her child is distressed (e.g., cries, fusses or whimpers).

70. Is so late in her responses, that it is not clear for the child what she is responding to.

71. Joins in the focus of her child's attention.

72. Notices when her child smiles and makes sounds.

73. When she is irritated with her child, she stops doing things with him/her.

74. Worries when her child tries new things, even when they are not dangerous.

75. Encourages her child to try new things.

76. Holds her child close to her to comfort him/her. 
77. Talks to her child regularly.

78. Plays games together with her child.

79. Becomes tense when her child needs a lot of attention.

80. Is annoyed if her child does not cooperate.

81. Clearly shows her child that she is happy with him/her.

82. Restricts her child's movements

83. Aloof/distant when doing things with her child.

84. The feelings that she shows do not match the feelings of the child, for example mother smiles when her child cries.

85. Suddenly interrupts things that she is doing with her child.

86. Stops physical contact before her child is contented.

87. Clearly opposes her child's wishes.

88. Often argues or disagrees with her child.

89. The way she handles her child makes her child content.

90. Is negative and hostile towards her child.

\section{Appendix B}

The final model can be specified as follows:

Level 1 (within cultural groups):

$$
\begin{aligned}
\mathrm{Y}_{\mathrm{ij}}= & \beta_{0 \mathrm{j}}+\beta_{10}\left(\text { income }_{\mathrm{ij}}\right)+\beta_{20}\left(\text { edum }_{\mathrm{ij}}\right)+\beta_{30}\left(\text { agem }_{\mathrm{ij}}\right) \\
& +\beta_{40}\left(\operatorname{nrchild}_{\mathrm{ij}}\right)+\beta_{50}\left(\operatorname{rural}_{\mathrm{ij}}\right)+\beta_{60}\left(\text { horind }_{\mathrm{ij}}\right) \\
& +\beta_{70}\left(\operatorname{vertind}_{\mathrm{ij}}\right)+\beta_{80}\left(\text { horcol }_{\mathrm{ij}}\right)+\beta_{90}\left(\operatorname{vertcol}_{\mathrm{ij}}\right) \\
& +\beta_{100}\left(\text { religion }_{\mathrm{ij}}\right)+\mathrm{e}_{\mathrm{ij}}
\end{aligned}
$$

Level 2 (between cultural groups):

$$
\beta_{0 \mathrm{j}}=\gamma_{00}+\mu_{0 \mathrm{j}}
$$

where $\gamma_{00}$ represents the overall intercept, and $\mu_{0 \mathrm{j}}$ represents the deviation of cultural groups to the overall intercept (intercept variance). 